\title{
Erratum
}

\section{The black hole binary nova Scorpii 1994 (GRO J1655-40): an improved chemical analysis ${ }^{\star}$}

\author{
J. I. González Hernández ${ }^{1,2,3}$, R. Rebolo ${ }^{3,4}$, and G. Israelian ${ }^{3}$ \\ 1 Observatoire de Paris-Meudon, GEPI, 5 place Jules Janssen, 92195 Meudon Cedex, France \\ e-mail: Jonay.Gonzalez-Hernandez@obspm.fr \\ ${ }^{2}$ CIFIST Marie Curie Excellence Team \\ 3 Instituto de Astrofísica de Canarias, 38205 La Laguna, Tenerife, Spain \\ e-mail: [rrl;gil]@iac.es \\ ${ }^{4}$ Consejo Superior de Investigaciones Científicas, Spain
}

A\&A 478, 203-217 (2008), DOI: 10.1051/0004-6361/20077141

Key words. black holes physics - stars: abundances - stars: individual: Scorpii 1994 (GRO J1655-40) - stars: supernovae: general $\mathrm{X}$-rays: binaries - errata, addenda

The values of the excitation potential, $\chi$, and oscillator strength, $\log g f$, of the three lines of the $\mathrm{Mg} \mathrm{Ib}$ triplet at 5167-83 $\AA$ in Table 2 are wrong. The correct values are:

- Mg I $15167.321 \AA ̊ 2: \chi=2.709 \mathrm{eV}$ and $\log g f=-1.03$

- Mg I $\lambda 5172.684 \AA: \chi=2.712 \mathrm{eV}$ and $\log g f=-0.40$

- Mg I $\lambda 5183.604 \AA: \chi=2.717 \mathrm{eV}$ and $\log g f=-0.18$.
All the synthetic spectra, as those displayed in Fig. 9, presented in the paper were computed using the correct values for the excitation potential and oscillator strengths of these Mg I lines. Therefore, although we made a mistake in writing the values of $\chi$ and $\log g f$ of these lines in Table 2, the Mg abundances presented in the paper are also correct.

^ Based on observations obtained with UVES at VLT Kueyen $8.2 \mathrm{~m}$ telescope in programme 073.D-0473(A). 